\title{
Epidemiology and Management Trends of Patients With Sepsis and Septic Shock in the Intensive Care Unit: A Prospective Trial in the Caribbean
}

Keevan Singh ${ }^{1,2}$, Seetharaman Hariharan ${ }^{1,3}$, Dale Ventour ${ }^{1,3}$, Deryk R. Chen ${ }^{3}$, Lorna G. Merritt-Charles 1,3 , Mark Sookwah ${ }^{2}$, Daynish Maharaj ${ }^{4}$, Sasha Sankar-Maharaj ${ }^{5}$

1. Anaesthesia and Intensive Care, University of the West Indies, St Augustine, TTO 2. Anaesthesia and Intensive Care, San Fernando General Hospital, San Fernando, TTO 3. Anaesthesia and Intensive Care, Eric Williams Medical Sciences Complex, Mt. Hope, TTO 4. Anaesthesia and Intensive Care, Port of Spain General Hospital, Port of Spain, TTO 5. Anaesthesia and Intensive Care, Sangre Grande Hospital, Sangre Grande, TTO

Corresponding author: Keevan Singh, keevansingh@gmail.com

\section{Abstract \\ Objectives}

To investigate the epidemiology, management, and predictors of mortality in severe sepsis and septic shock in the intensive care units (ICUs) of Trinidad, Trinidad \& Tobago.

\section{Methods}

A prospective observational study in four ICUs over a one-year period (August 2017-August 2018) was conducted. Physiologic variables, treatment data, and outcomes were collected on admission to ICU and daily until 28 days. The 28-day mortality and ICU mortality were recorded. Subgroup analysis was performed based on survival, and predictors of mortality were determined through logistic regression.

\section{Results}

Outcome data were available for 163 patients. The 28-day mortality rates for sepsis and septic shock were $42 \%$ and $47 \%$, respectively. ICU mortality rate for sepsis was $34 \%$. The most common suspected source of infection was pneumonia (33\%). Acute kidney injury (AKI) was common and present in $71 \%$ of patients, with renal replacement therapy only being used in $30 \%$ of cases. Mechanical ventilation was required in $84 \%$ of cases. Moderate-to-severe acute respiratory distress syndrome (ARDS) (OR: 4; 95\% CI: 1.9-8.8; $<<0.001$ ) and the development of AKI (all stages) (OR: 10; 95\% CI: 3.9-30.2; p < 0.001) were found to be predictive of mortality. Incidence of mechanical ventilation, moderate-to-severe ARDS, stage 3 AKI, septic shock, and failure to achieve a mean arterial pressure of $>60 \mathrm{mmHg}$ within the first 24 hours of admission were higher in patients who did not survive $(\mathrm{p}<0.05)$.

Review began 09/09/2020 Review ended 10/10/2020 Published 10/16/2020

\section{() Copyright 2020}

Singh et al. This is an open access article distributed under the terms of the Creative Commons Attribution License CC-BY 4.0., which permits unrestricted use, distribution, and reproduction in any medium, provided the original author and source are credited.

\section{Conclusions}

Sepsis and septic shock are associated with a high 28-day mortality. Organ dysfunction with renal and pulmonary involvement was an important factor in predicting a higher mortality.

Categories: Internal Medicine, Infectious Disease

Keywords: sepsis, septic shock, icu, sofa, ards, aki, outcomes, mortality

\section{Introduction}

Over the last decade, there have been several innovations in the diagnosis and management of sepsis and septic shock [1,2]. Current definitions focus on the detection of organ dysfunction instead of inflammatory parameters, with sepsis now defined as a dysregulated host response rather than a purely inflammatory condition [2]. However, despite these advances, there remains a significant global burden of disease, with 49 million cases of sepsis and 11 million deaths being reported worldwide in 2017 [3].

Stevenson et al. using control arm data from randomized clinical trials showed a mortality rate of up to $30 \%$ for severe sepsis [4]. In developing and low-resource countries, which may not have been represented by trial data, the mortality rate for sepsis is even higher, with an almost $50 \%$ mortality rate being reported for severe sepsis and septic shock in developing and resource-poor countries [5-8].

The epidemiology of sepsis has been well studied in developed countries, with most guidelines being developed based on these populations [8]. Sepsis in low-resource settings has its own unique epidemiological and therapeutic challenges, hence the need for a more global focus in sepsis [9]. As sepsis 
remains a significant global disease, data from developing and low-resource countries can add global insight into the study of this disease process.

Investigators from many resource-limited countries have published data regarding the epidemiology and outcomes of sepsis in their regions [3-5]. In a single emergency department cohort in Jamaica, where 117 patients with severe sepsis and septic shock were studied, a mortality rate of $25 \%$ was observed [10]. To date, there has been no consistent published data on sepsis and sepsis outcomes in a Caribbean intensive care unit (ICU) setting.

Trinidad \& Tobago is a twin-island republic lying close to the South America mainland with a population of approximately 1.5 million and a diverse ethnic population. Although ranked as a high-income country according to the World Bank, there are currently only two adult ICU beds per 100,000 persons locally. In developing countries, such as ours, prioritized healthcare spending can present problems for critically ill patients. Globally, many patients with sepsis and septic shock require ICU level care. Hence, the burden of sepsis on countries with low ICU bed numbers is significant. This can thus pose significant public health concerns to healthcare resource-poor countries.

The aim of our study is to investigate the 28-day mortality rate of severe sepsis and septic shock in Trinidadian ICUs. The severity scores, location of the septic source, treatment trends, and factors associated with mortality will also be described in our population.

\section{Materials And Methods}

This study was conducted as a prospective observational trial in the four local ICUs, San Fernando General Hospital (SFGH), Eric Williams Medical Sciences Complex (EWMSC), Port of Spain General Hospital (POSGH), and Sangre Grande Hospital (SGH). The trial was run over a one-year period from August 2017 to August 2018.

Approvals were obtained from the Campus Research Ethics Committee, The University of the West Indies, St Augustine, and the Ethics Committees of each of the four participating hospitals. The respective ethics committees, due to the observational nature of the study, waived requirements for written informed consent. Data collected were limited to only that which formed part of the routine clinical management of intensive care patients.

\section{Patients}

All patients older than 18 years admitted to the ICU and fitting the criteria for sepsis and septic shock during the study period were eligible for inclusion.

Exclusion criteria included patients who presented with a primary diagnosis of hemodynamic instability secondary to ongoing bleeding, burns, acute coronary syndrome, acute major cerebrovascular event, acute pulmonary edema, seizures, drug overdose, and major cardiac arrhythmias.

\section{Definitions}

Sepsis was defined according to the Third International Consensus Definition for Sepsis and Septic Shock (Sepsis-3): suspected or confirmed infection and a change in Sequential Organ Failure Assessment (SOFA) score $\geqslant 2[2]$. Patients who were not known to have any organ dysfunction were listed as having a baseline SOFA of 0 . Septic shock was diagnosed in patients with sepsis and persistent hypotension requiring vasopressors to maintain a MAP of $\geqslant 65 \mathrm{mmHg}$, despite adequate fluid resuscitation and evidence of tissue hypoperfusion. As lactate was not readily available, base excess $\leqslant 4 \mathrm{mmol} / \mathrm{L}$ was used as evidence of tissue hypoperfusion when diagnosing septic shock $[2,11,12]$.

Acute respiratory distress syndrome (ARDS) was diagnosed according to Berlin criteria, and KDIGO (Kidney Disease Improving Global Outcomes) criterion was used for diagnosis and staging of acute kidney injury (AKI) $[13,14]$.

\section{Data collection}

All ICU admissions were screened by team members at the various hospitals for possible inclusion. Data were obtained from the patients' notes using a manual data collection instrument. This instrument was frequently inspected by the individual clinical leads to ensure the accuracy and completeness of data entry. Data collected included demographics, suspected source of sepsis, monitoring used, management trends, and outcome data that included length of stay in ICU, days on mechanical ventilation, and survival. Clinical data were collected for calculating maximum SOFA and Acute Physiology and Chronic Health Evaluation II (APACHE II) scores in the first 24 hours of admission to the ICU. Mean arterial pressure (MAP) was recorded hourly for the first 24 hours and used to determine the mean MAP over the first 24 hours. Patients were also followed daily during their ICU stay for the development of ARDS and AKI. Mortality was recorded in ICU as well as at 28 days after ICU admission. 


\section{Cureus}

\section{Analysis}

Data analysis was performed using Statistical Package for Social Sciences (SPSS) Version 20 (IBM Corp., Armonk, NY, USA). Normally distributed data were presented as mean and standard deviations (SD), data which were not normally distributed were presented as median and interquartile ranges (IQRs), and categorical data were presented as proportions. Continuous normally distributed data were analyzed using Student's t-test and non-normally distributed data were analyzed using the Mann-Whitney U test. Categorical data were analyzed using the chi-square or Fisher exact test, as appropriate.

Multivariate logistic regression was used to determine the factors associated with 28-day and ICU survival. Predictors were chosen based on their reported predictive ability, use in other predictive models, current clinical usage, and perceived importance. The predictor and outcome variables were analyzed with a stepwise approach using a backward elimination method based on the likelihood ratio (backward LR). Mortality predictors used in the regression analysis included APACHE II and SOFA scores, age, MAP $<60$ mmHg within the first 24 hours, base excess, presence of septic shock, days on mechanical ventilation, and development of moderate-to-severe ARDS or AKI during their ICU course. A p-value of $<0.05$ was considered statistically significant.

\section{Results}

On admission to ICU, 183 patients were suspected of having severe sepsis. Out of these, 163 persons met the inclusion criteria and were included in the study. Outcome data were available for all 163 persons, and they were included in the analysis.

\section{Demographics}

The overall demographics are presented in Table 1.

\begin{tabular}{|c|c|c|c|c|}
\hline Demographics & All patients $(n=163)$ & Survivors $(\mathbf{n}=95)$ & Non-survivors $(n=68)$ & p-Values* \\
\hline Male, n (\%) & $82(50)$ & $37(47)$ & $45(54)$ & 0.43 \\
\hline Female, n (\%) & $81(50)$ & $50(53)$ & $31(38)$ & 0.43 \\
\hline Age, median (IQR) & $52(39-65)$ & $50(39-61)$ & $54(40-71)$ & 0.07 \\
\hline \multicolumn{5}{|l|}{ Ethnicity, $\mathrm{n}(\%)$} \\
\hline Afro-Trinidadian & $43(26.4)$ & $24(25)$ & $19(28)$ & 0.72 \\
\hline Indo-Trinidadian & $108(66.3)$ & $66(70)$ & $42(62)$ & 0.32 \\
\hline Mixed/other & $12(7.4)$ & $5(5)$ & $7(10)$ & 0.24 \\
\hline \multicolumn{5}{|l|}{ Hospital, n (\%) } \\
\hline Hospital 1 & $101(62)$ & $41(60)$ & $60(63)$ & 0.745 \\
\hline Hospital 2 & $26(26)$ & $17(17.9)$ & $9(13.2)$ & 0.52 \\
\hline Hospital 3 & $20(20)$ & $9(9.5)$ & $11(16.2)$ & 0.23 \\
\hline Hospital 4 & $16(9.8)$ & $9(9.5)$ & $7(10.3)$ & 0.86 \\
\hline
\end{tabular}

\section{TABLE 1: Demographic factors of study population}

* $p$-Values were derived using chi-square analysis, Fisher exact test, and Mann-Whitney test as appropriate.

IQR, interquartile range

Gender was evenly split, with males and females consisting of $50 \%$ of the population. Persons of East Indian descent (Indo-Trinidadian) comprised the largest proportion of patients (108 [66\%]) followed by those of African descent (Afro-Trinidadian) (43 [26\%]). The median age of the population was 52 years (range: $39-65$ years). The majority of cases came from one hospital, which accounted for 101 (62\%) cases.

\section{Characteristics}

The descriptive clinical variables are depicted in Table 2 . 


\section{Cureus}

\begin{tabular}{|c|c|c|c|c|}
\hline Characteristics median (IQR) & All patients $(n=163)$ & Survivors $(n=95)$ & Non-survivors $(n=68)$ & p-Values \\
\hline SOFA & $8(6-10)$ & $8(5-10)$ & $9(7-1)$ & 0.03 \\
\hline APACHE II & $17(13-22)$ & $15(12-20)$ & $20(16-27)$ & $<0.001$ \\
\hline Mechanically ventilated $(\%)$ & $137(84)$ & $73(77)$ & $64(94)$ & $<0.001$ \\
\hline Base excess & $-9(-12$ to -5$)$ & $-8(-11$ to -5$)$ & $-11(-13$ to -6$)$ & $<0.001$ \\
\hline AKI (any stage), n (\%) & $115(71)$ & $53(56)$ & $62(91)$ & $<0.001$ \\
\hline AKIN stage 1 & $7(4.3)$ & $3(3)$ & $4(6)$ & 0.45 \\
\hline AKIN stage 2 & $31(19)$ & $15(15.8)$ & $16(24)$ & 0.23 \\
\hline AKIN stage 3 & $77(47)$ & $35(37)$ & $42(62)$ & $<0.001$ \\
\hline RRT (\%) & $48(30)$ & $23(48)$ & $25(52)$ & 0.08 \\
\hline Moderate-to-severe ARDS (\%) & $74(45)$ & $32(43)$ & $42(57)$ & $<0.001$ \\
\hline Septic shock (\%) & $119(73)$ & $63(66)$ & $56(82)$ & 0.03 \\
\hline MAP < 60 mmHg (\%) & $12(7.4)$ & $2(2)$ & $10(15)$ & 0.00 \\
\hline Days on ventilator & $4(1-8)$ & $4(1-7)$ & $5(2-11)$ & 0.02 \\
\hline ICU days & $6(3-11)$ & $6(4-10)$ & $6(3-13)$ & 0.96 \\
\hline \multicolumn{5}{|l|}{ Suspected source (\%) } \\
\hline Lungs & $54(33)$ & $34(36)$ & $20(29)$ & 0.40 \\
\hline Urological & $23(14)$ & $19(20)$ & $4(6)$ & 0.01 \\
\hline Abdomen & $27(17)$ & $10(11)$ & $17(25)$ & 0.02 \\
\hline Pancreas & $10(6)$ & $6(6)$ & $4(6)$ & 1.00 \\
\hline CNS & $6(4)$ & $2(2)$ & $4(6)$ & 0.23 \\
\hline Skin/soft tissue & $9(5)$ & $5(5)$ & $4(6)$ & 1.00 \\
\hline Surgical site & $5(3)$ & $1(1)$ & $4(6)$ & 0.16 \\
\hline Other/unknown & 29 (18) & 18 & $11(16)$ & 0.68 \\
\hline
\end{tabular}

\section{TABLE 2: Patient characteristics and sepsis-related organ involvement}

ARDS, acute respiratory distress syndrome; APACHE II, Acute Physiology and Chronic Health Evaluation II; AKI, acute kidney injury; AKIN, Acute Kidney Injury Network; CNS, central nervous system; ICU, intensive care unit; IQR, interquartile range; MAP, mean arterial pressure, RRT, renal replacement therapy; SOFA, Sequential Organ Failure Assessment

Median (IQR) admission SOFA and APACHE II scores were 8 (6-10) and 17 (13-22), respectively. The majority of patients, 119 (73\%), had septic shock. A total of 115 (71\%) patients developed AKI, of which 77 (43\%) developed stage $3 \mathrm{AKI}$. Renal replacement therapy (RRT) was only used in 48 (30\%) patients. Moderate-tosevere ARDS was present in 74 (45\%) patients, and the respiratory tract (lungs) was found to be the most commonly affected source of infection (54 [33\%]). Mechanical ventilation was required in 137 (84\%) patients.

No specific source could be found in $18 \%$ of patients. An abdominal and urological source was found in $17 \%$ and $14 \%$ of patients, respectively.

\section{Management parameters}

The management variables are shown in Table 3. 


\section{Cureus}

\begin{tabular}{|c|c|}
\hline Management parameter, $\mathrm{n}(\%)$ & $N=163$ \\
\hline Arterial line & $160(98)$ \\
\hline CVP line & $147(90)$ \\
\hline Blood culture within 24 hours & $139(85)$ \\
\hline Average MAP > $60 \mathrm{mmHg}$ within 24 hours & $152(93)$ \\
\hline Positive culture obtained & $21(13)$ \\
\hline \multicolumn{2}{|l|}{ Vasopressor choice } \\
\hline None & $41(25)$ \\
\hline Noradrenaline & $69(42)$ \\
\hline Dopamine & $3(2)$ \\
\hline Noradrenaline and adrenaline & $33(20)$ \\
\hline Noradrenaline and dobutamine & $3(2)$ \\
\hline Other & $14(9)$ \\
\hline \multicolumn{2}{|l|}{ Antibiotic choice } \\
\hline Meropenem/imipenem & $41(25)$ \\
\hline Piperacillin-tazobactam & $54(33)$ \\
\hline Third-generation cephalosporin (ceftriaxone, cefuroxime, cefotaxime) & $35(22)$ \\
\hline Levofloxacin & 7 (4) \\
\hline Other (monotherapy) & $7(4)$ \\
\hline mbination therar & $19(1$ \\
\hline
\end{tabular}

\section{TABLE 3: Management parameters of patient population}

CVP, central venous pressure; MAP, mean arterial pressure

Most patients were managed with arterial (98\%) and central lines (90\%). A mean MAP of > $60 \mathrm{mmHg}$ in the first 24 hours of admission was achieved in 152 (93\%) patients. Noradrenaline was the most common vasopressor used in 69 (42\%) patients, and this was followed by the noradrenaline-adrenaline combination in 33 (20\%) patients (Table 3). Positive cultures were only obtained in 21 (13\%) patients. Escherichia coli was the most common organism cultured (19\%) followed by Acinetobacter (14\%) and Klebsiella (14\%).

Piperacillin-tazobactam was used in $33 \%$ of patients followed by carbapenems (meropenem/imipenem) in $25 \%$.

\section{Outcomes}

The ICU mortality rate was $34 \%$, whereas the 28 -day mortality rate for sepsis was $42 \%$. Patients with septic shock had a 28 -day mortality rate of 47\%. Patients spent a median of 6 days (range: 3-11 days) in the ICU with 4 days (range: 1-8 days) on a mechanical ventilator. There was no statistical difference in outcomes based on admitting hospital.

\section{Comparison based on 28-day survival}

The variables were compared between survivors and non-survivors at 28 days, which is shown in Table 2 . Non-survivors had significantly higher SOFA $(\mathrm{p}=0.03)$ and APACHE II $(\mathrm{p}<0.001)$ scores on admission than survivors. Base excess was significantly lower in non-survivors $(p<0.001)$. The mortality was also higher in patients requiring mechanical ventilation compared to those who did not require this intervention ( $94 \% \mathrm{vs}$ $77 \%, \mathrm{p}<0.001$ ). Incidence of moderate-to-severe ARDS, stage 3 AKI, septic shock, and a failure to achieve a mean MAP of $>60 \mathrm{mmHg}$ was significantly higher in patients who did not survive $(\mathrm{p}<0.05)$. Urological sepsis was more common in survivors $(\mathrm{p}=0.01)$, whereas abdominal sepsis was more common in patients who died $(\mathrm{p}=0.02)$. 


\section{Cureus}

\section{Comparison based on ICU survival}

Significant differences between groups were similar to trends seen in 28-day survival (Table 2). APACHE II score, SOFA score, base excess, stage 3 AKI, moderate-to-severe ARDS, septic shock, and urological and abdominal sepsis were all significantly different between ICU survivors and non-survivors. The incidence of RRT was higher in patients who died in ICU $(41 \%$ vs $23 \%$; $p=0.02)$.

\section{Predictors of 28-day mortality}

In the logistic regression analysis (Table 4), out of the initial predictors and using the backward LR approach, three variables (any stage of AKI, moderate-to-severe ARDS, and low MAP) were included as the final predictors for 28-day mortality and four variables for ICU mortality (any stage AKI, moderate-to-severe ARDS, low MAP, base excess).

\begin{tabular}{|c|c|c|}
\hline Predictor variable for 28-day outcome & OR (95\% CI) & p-Value \\
\hline Apache II & $1.1(0.98-1.10)$ & 0.16 \\
\hline SOFA score & $0.9(0.70-1.06)$ & 0.22 \\
\hline Age & $1.0(0.97-1.00)$ & 0.96 \\
\hline Ventilator days & $1.0(0.97-1.10)$ & 0.30 \\
\hline Septic shock & $1.0(0.30-2.95)$ & 0.92 \\
\hline Base excess & $1.0(0.88-1.05)$ & 0.41 \\
\hline Moderate-to-severe ARDS ${ }^{\star}$ & $4.0(1.89-8.83)$ & $<0.001$ \\
\hline Low MAP* & $6.1(0.94-40.33)$ & 0.06 \\
\hline Any AKI ${ }^{*}$ & $10.9(3.93-30.17)$ & $<0.001$ \\
\hline
\end{tabular}

\section{TABLE 4: Predictor variables and its effect on 28-day outcome in the logistic regression analysis}

* Represents the variables that were included in the final analysis using the backward LR method.

ARDS, acute respiratory distress syndrome; AKI, acute kidney injury; LR, likelihood ratio; MAP, mean arterial pressure; SOFA, Sequential Organ Failure Assessment

In the logistic regression analysis, the only factors that were significantly associated with 28-day mortality were the presence of moderate-to-severe ARDS (OR: 4; 95\% CI: 1.9-8.8; $\mathrm{p}<0.001$ ) and the development of AKI (any stage, OR: 10; 95\% CI: 3.9-30; $<<0.001$ ). AKI and moderate-to-severe ARDS were also associated with ICU mortality $(\mathrm{p}<0.01)$.

\section{Discussion}

Sepsis has received considerable attention over the last decade with an attendant increase in awareness of its presentation and need for emergent management. Global initiatives such as the Surviving Sepsis Campaign (SSC) have sought to improve the quality of care for these patients by advocating protocolized care and implementing evidence and best practice-based recommendations to guide clinicians in the early diagnosis, monitoring, and management of the disease [1]. Adherence to these recommendations and performance bundles, even in low-resource and middle-income countries, has been shown to reduce the odds of hospital mortality [15].

When the management parameters in the patients of the present study are considered (Table 3), it can be noted that the majority of patients had arterial and central venous pressure monitoring and more than $90 \%$ were able to obtain a MAP > $60 \mathrm{mmHg}$ within the first 24 hours. Empiric broad-spectrum antibiotics were also frequently used, and a blood culture was performed within 24 hours in $85 \%$ of patients. Noradrenaline was also the most commonly used vasopressor (42\%). These findings illustrate that clinicians are aware of the standard management guidelines for sepsis. However, the challenge exists in translating this knowledge to consistent practice to improve outcomes, which from time to time may be significantly limited in a resource-constrained healthcare system. We also note that only $13 \%$ of patients had positive cultures, and this may have been due to the fact that most cultures were taken late and after antibiotics were started. 
States, United Kingdom, and Australia have shown no difference between this protocolized care and routine care groups [16-18]. However, all the standard care groups received a high level of care including prompt resuscitation similar to that advocated by SSC. Despite this foundation for clinical management of sepsis, the challenge still exists in knowing which practices are feasible and cost-efficient to adopt in low-resource countries [9].

The median age of the patient cohort in the present study was 52 years, which represented a relatively younger cohort of affected patients compared to those of more developed countries, where patients generally tend to have been a decade older $[16,18]$. Healthcare initiatives in this patient group can have a meaningful impact on society and may justify an increased critical care spending to improve outcomes. Loss of these relatively younger patients from sepsis in low-resource settings can have a far-reaching economic impact on the affected families [9].

Shortages of ICU beds are a significant problem in sepsis management. This arises due to the intensity of management advocated in guidelines and the need for organ support, which can only be found in ICUs. Sufficient ICU beds in many low-resource countries pose a dilemma especially when there may be other pressing healthcare needs [9]. The majority (84\%) of the patients in this study needed mechanical ventilation and 73\% required vasopressor infusions, making ICU admission and critical care management mandatory. In comparison, a study published from Jamaica, another Caribbean country, showed that although 113 patients were diagnosed to have severe sepsis in the emergency department, only one patient could be admitted to the ICU [10]. This highlights the ICU-bed deficiencies plaguing low-resource settings.

The median admission SOFA and APACHE II scores were 8 (range: 6-10) and 17 (range: 13-22), and the overall predicted mortality was $33 \%$ and $25 \%$, respectively. This may indicate that the intensity of the illness was severe in this cohort of the patients on presentation to the ICU. This was comparable to the severity of illness scores reported in many recently reported sepsis trials where admission SOFA scores ranged from 2 (United Kingdom) to 8 (Japan, India) and APACHE II scores ranged from 15 (Australia) to 21 (United States) [7,16-19].

The 28-day mortality rate for sepsis and septic shock was $42 \%$ and $47 \%$, respectively, and both these are comparatively higher than those reported in recent trials. However, many of these reports were from highincome developed countries, and the mortality (60 and 90 days) ranged from 18-30\% [16-18]. When considering global data, the in-hospital mortality rate due to sepsis ranges from 40 to $60 \%$ [8]. In the present study, there was also a notable difference between ICU mortality (34\%) and 28-day mortality. It is likely that after discharge from ICU to the general wards, the level of care provided to such patients was not commensurate with the unique challenges faced by such patients. This could have led to an increase in mortality as seen. Low ICU bed numbers would also increase the pressure on clinicians for early discharge in a patient who may still require a higher level of care than could be provided in the general wards. Quality improvement measures in sepsis for low-resource countries should aim to provide a consistent level of care for these patients, not only in the emergency departments and ICUs but also in general medical and surgical wards. Failure to do this can lead to wasted resources spent in the ICU, with no overall beneficial impact of ICU care on patient mortality.

The presence of moderate-to-severe ARDS $(\mathrm{OR}=4)$ and the development of any stage $\mathrm{AKI}(\mathrm{OR}=10)$ were significantly associated with 28-day mortality in the present study. Both these conditions require significant critical care expertise, and in the case of AKI, additionally, significant resources are needed. This can be inferred from the fact that AKI was diagnosed in $71 \%$ of patients, whereas only $30 \%$ received RRT. The sole modality used for RRT was hemofiltration, which was only performed in one ICU of all the four ICUs studied. The high cost of replacement fluids is an important consideration in this regard. Poor access to RRT in patients with AKI is a recognized problem and has been reported from low- and middle-income countries [20].

Pneumonia was the most commonly identified source of sepsis (33\%), with gram-negative organisms being the most commonly cultured. Almost half of all the study patients developed moderate-to-severe ARDS (45\%). ARDS was a 28-day mortality predictor in the present study, which follows the trend of high global ICU mortality rate associated with ARDS. In the LUNG-SAFE (Large observational study to UNderstand the Global impact of Severe Acute respiratory FailurE) study using an international cohort, moderate and severe ARDS was associated with a mortality rate of $40 \%$ and $46 \%$ respectively, similar to that of the present study [21]. The authors noted that ARDS was largely unrecognized and identified several shortcomings in clinical management, which could have been true in our setting also.

There were several limitations to our study. The total number of patients admitted to the ICUs during the period was not recorded, and hence the overall incidence of sepsis could not be determined. One ICU represented $63 \%$ of cases; hence, there is a possibility that the incidence of sepsis might have been underreported in the other ICUs. No data were available on patients' conditions before ICU admission and the lead-time from the identification of sepsis to ICU admission, which may have further affected the outcomes. 
local availability, base excess was used as a surrogate for lactate when septic shock was diagnosed [11]. This may have led to some patients' misclassification as septic shock due to the imperfect relation between lactate and base excess. However, the majority of patients classified as septic shock (97\%) were on moderately high doses of vasopressors, making the diagnosis likely.

\section{Conclusions}

In conclusion, this study was able to describe the epidemiology and management trends for sepsis in four ICUs in Trinidad. Sepsis and septic shock are associated with high mortality rates, and mortality is also higher after discharge from ICU. This study will provide basal data from a Caribbean country regarding sepsis and its management, which will be useful for quality improvement measures including resource allocation.

\section{Additional Information \\ Disclosures}

Human subjects: Consent was obtained by all participants in this study. University of The West Indies Campus Ethics Committee issued approval CEC 159/03/17. Approval was granted by the University of the West Indies Campus Ethics Committee and the respective ethics committees of each of the various participating hospitals for the conduct of this trial. The ethics committees, due to the observational nature of the study, waived requirements for written informed consent. Data collected were limited to only that which formed part of the routine clinical management of intensive care patients. . Animal subjects: All authors have confirmed that this study did not involve animal subjects or tissue. Conflicts of interest: In compliance with the ICMJE uniform disclosure form, all authors declare the following: Payment/services info: All authors have declared that no financial support was received from any organization for the submitted work. Financial relationships: All authors have declared that they have no financial relationships at present or within the previous three years with any organizations that might have an interest in the submitted work. Other relationships: All authors have declared that there are no other relationships or activities that could appear to have influenced the submitted work.

\section{Acknowledgements}

We thank the data collection teams at the various hospitals for their hard work and assistance in this trial. SFGH: Dr. Tameem Mohammed, Dr. Dayna Ramkeesoon, Dr Nickilia Maurice. POSGH: Dr Sasha Hinds. SGH: Dr Marishka DePeiza.

\section{References}

1. Rhodes A, Evans LE, Alhazzani W, et al.: Surviving Sepsis Campaign: international guidelines for management of sepsis and septic shock: 2016. Intensive Care Med. 2017, 43:304-377. 10.1007/s00134-0174683-6

2. Singer M, Deutschman CS, Seymour CW, et al.: The Third International Consensus Definitions for Sepsis and Septic Shock (Sepsis-3). JAMA. 2016, 315:801-810. 10.1001/jama.2016.0287

3. Rudd KE, Johnson SC, Agesa KM, et al.: Global, regional, and national sepsis incidence and mortality: analysis for the Global Burden of Disease Study. Lancet. 2020, 395:200-211. 10.1016/S0140-6736(19)329897

4. Stevenson EK, Rubenstein AR, Radin GT, Wiener RS, Walkey AJ: Two decades of mortality trends among patients with severe sepsis: a comparative meta-analysis. Crit Care Med. 2014, 42:625-631. 10.1097/ccm.0000000000000026

5. Silva E, Pedro MdA, Sogayar ACB, et al.: Brazilian Sepsis Epidemiological Study (BASES study) . Crit Care. 2004, 8:251. 10.1186/cc2892

6. Rodríguez F, Barrera L, De La Rosa G, et al.: The epidemiology of sepsis in Colombia: a prospective multicenter cohort study in ten university hospitals. Crit Care Med. 2011, 39:1675-1682. 10.1097/CCM.0b013e318218a35e

7. Paary TT, Kalaiselvan MS, Renuka MK, Arunkumar AS: Clinical profile and outcome of patients with severe sepsis treated in an intensive care unit in India. Ceylon Med J. 2016, 61:181-184. 10.4038/cmj.v61i4.8386

8. Cecconi M, Evans L, Levy M, Rhodes A: Sepsis and septic shock. Lancet. 2018, 392:75-87. 10.1016/S01406736(18)30696-2

9. Schultz MJ, Dunser MW, Dondorp AM, et al.: Current challenges in the management of sepsis in ICUs in resource-poor settings and suggestions for the future. Intensive Care Med. 2017, 43:612-624. 10.1007/s00134-017-4750-z

10. Edwards R, Hutson R, Johnson J, Sherwin R, Gordon-Strachan G, Frankson M, Levy P: Severe sepsis in the emergency department - an observational cohort study from the university hospital of the West Indies. West Indian Med J. 2013, 62:224-229. 10.7727/wimj.2012.194

11. Montassier E, Batard E, Segard J, Hardouin JB, Martinage A, Le Conte P, Potel G: Base excess is an accurate predictor of elevated lactate in ED septic patients. Am J Emerg Med. 2012, 30:184-187. 10.1016/j.ajem.2010.09.033

12. Shankar-Hari M, Phillips GS, Levy ML, et al.: Developing a new definition and assessing new clinical criteria for septic shock: for the Third International Consensus Definitions for Sepsis and Septic Shock (Sepsis-3). JAMA. 2016, 315:775-787. 10.1001/jama.2016.0289

13. Ranieri VM, Rubenfeld GD, Thompson BT, et al.: Acute respiratory distress syndrome: the Berlin definition . JAMA. 2012, 307:2526-2533. 10.1001/jama.2012.5669 


\section{Cureus}

14. Kellum JA, Lameire N, Aspelin P, et al.: Kidney disease: improving global outcomes (KDIGO) acute kidney injury work group. KDIGO clinical practice guideline for acute kidney injury. Kidney Int Suppl. 2012, 2:1138.

15. Levy MM, Rhodes A, Phillips GS, et al.: Surviving Sepsis Campaign: association between performance metrics and outcomes in a 7.5-year study. Crit Care Med. 2015, 43:3-12. 10.1097/ccm.0000000000000723

16. The ProCESS Investigators: A randomized trial of protocol-based care for early septic shock . N Engl J Med. 2014, 370:1683-1693. 10.1056/NEJMoa1401602

17. The ARISE Investigators and the ANZICS Clinical Trials Group: Goal-directed resuscitation for patients with early septic shock. N Engl J Med. 2014, 371:1496-1506. 10.1056/NEJMoa1404380

18. Mouncey PR, Osborn TM, Power GS, et al.: Trial of early, goal-directed resuscitation for septic shock . N Engl J Med. 2015, 372:1301-1311. 10.1056/NEJMoa1500896

19. Ogura H, Gando S, Saitoh D, et al.: Epidemiology of severe sepsis in Japanese intensive care units: a prospective multicenter study. J Infect Chemother. 2014, 20:157-162. 10.1016/j.jiac.2013.07.006

20. Olowu WA, Niang A, Osafo C, et al.: Outcomes of acute kidney injury in children and adults in sub-Saharan Africa: a systematic review. Lancet Glob Health. 2016, 4:242-250. 10.1016/s2214-109x(15)00322-8

21. Bellani G, Laffey JG, Pham T, et al.: Epidemiology, patterns of care, and mortality for patients with acute respiratory distress syndrome in intensive care units in 50 countries. JAMA. 2016, 315:788-800. 10.1001/jama.2016.0291 